\title{
Contribution of the Dual Mobility Acetabulum in the Treatment of Trochanteric Fracture in the Elderly
}

ISSN: 2576-8875

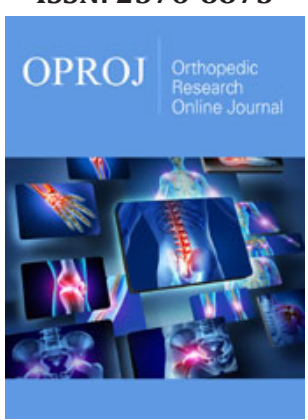

*Corresponding author: Pr. Ait El Hadj L, Department of Orthopaedic Surgery, EHS Benaknoun, Algeria

Submission: 制 December 11, 2019

Published: 眥December 16, 2019

Volume 6 - Issue 2

How to cite this article: Ait El Hadj L, Touati A, Fourmas S, Yakoubi M. Contribution of the Dual Mobility Acetabulum in the Treatment of Trochanteric Fracture in the Elderly. Ortho Res Online J. 6(2). OPROJ.000635.2019.

DOI: 10.31031/OPROJ.2019.06.000635

Copyright@: Pr. Ait El Hadj L, This article is distributed under the terms of the Creative Commons Attribution 4.0 International License, which permits unrestricted use and redistribution provided that the original author and source are credited.

\author{
Pr. Ait El Hadj L ${ }^{1 *}$, Touati $\mathrm{A}^{2}$, Fourmas $\mathrm{S}^{2}$ and Pr. Yakoubi $\mathrm{M}^{3}$ \\ ${ }^{1}$ Department of Orthopaedic Surgery, Algeria \\ ${ }^{2}$ EHS Benaknoun, Algeria \\ ${ }^{3}$ Head of Department, EHS Benaknoun, Algeria
}

\begin{abstract}
It appears that trochanteric fractures are always a challenge, as the risk of mortality at 1 year and the rates of complications are considerably high. During the last 20 years, various hardware improvements have been made, with the same goal: to obtain sufficient stability of the assembly to allow the resumption of support as early as possible. Sometimes, the consolidation is lacking causing serious functional damage in the elderly. Total hip arthroplasty has been advocated as a recovery procedure after treatment failure. We initiated a work in the main objective and to give back to the old subject any possibility of recovering as quickly as possible his autonomy. 22 patients benefitted from a prosthetic replacement, 12 of first intention and 10 after failure of conservative treatment. The acetabular cups were cemented with 2 retentive cups, 18 dual-mobility and 02 standard. Implantation of the prosthesis took an average of 45 minutes. One patient died during hospitalization, another patient within 90 days. The results were very good and good for the other patients. 2 dislocations occurred postoperatively. The publications on this subject generally report only a very limited number of cases and are few in number. Our study shows that total hip arthroplasty is a reliable technique to treat the complications of osteosynthesis of fractures per and under -trochanteric in the elderly. The complexity of the fracture associated with other factors related to the patient himself may lead the surgeon to consider arthroplasty as the treatment of choice.
\end{abstract}

Keywords: Trochanteric fractures; Total hip arthroplasty; Dual-mobility cups

\section{Introduction}

Trochanteric fractures are a major cause of mortality and mortality in the elderly. The incidence of all age fractures is around 80 per 100,000 people, with a significant financial burden. The optimal treatment for trochanteric fractures remains controversial [1]. It seems that trochanteric fractures are still a challenge, a risk of mortality at 1 year and a high complication rate compared to fractures of the femoral neck. During the last 20 years, various hardware improvements have been made, with the same goal: to obtain sufficient stability of the assembly to allow the resumption of support as early as possible. But whatever the quality and strength of the osteosynthesis material, the real problem is, in fact, in the old men, the quality of the bone in which it is implanted and the characteristics of the fracture. However, in view of the frequency of early mechanical failures and the caution of post-osteosynthesis management, several authors proposed hip arthroplasty in the 1980s [2]. Like cervical fractures, the goal is to give patients the best chance of having the fastest possible follow-up for resumption of support, return to the previous structure of life and recovery of maximum autonomy. There are two types of argumentation for the treatment of trochanteric fractures by arthroplasty: the unstable nature of certain types of fractures and the quality of the bone. Osteosynthesis remains difficult regardless of the strength of the implant and the context of the operated (age, associated defects) that makes it desirable to give him every opportunity to regain his autonomy as soon as possible and return to his life structure earlier. The aim of this study is to compare the short-term results of a prospective series of trochanteric fractures treated with total hip arthroplasty, either after failure of conservative treatment or immediately [3]. 


\section{Material and Methods}

After studying the literature, several factors were studied such as age, sex, poor bone quality and osteoarthritis of the hip. We have initiated a work with the main objective is to give back to the old subject any possibility of recovering as quickly as possible his autonomy and to return to his previous structure of life. Twenty-two patients benefited from prosthetic replacement, 12 from first intention and 10 after failure of conservative treatment. We operated on 22 patients, 14 men and 08 women (sex ratio of 1.4 man for one woman). The average age was 79 years old with a minimum of 75 years and a maximum of 92 years. The comorbidities observed preoperatively are summarized in Table 1. The retained fractures, of which the so-called unstable fractures are: 10 (A2) and 5 (A3), 7 (A1) with associated hip osteoarthritis. The mean preoperative Parker score was 5.3 with extremes of (4.9). Twelve of our patients received first-line prosthesis (Figure1), 08 after failure of conservative treatment (Figure 2), 5 nail-plates, 3 Gamma nails and 2 therapeutic discontinuations. The reason for the reoperation was in 5 cases disassembly of the osteosynthesis before the $3^{\text {rd }}$ month postoperative (Figure 3). In 03 cases, it was a late reoperation, beyond the third postoperative month due to nonunion Sixty percent of our patients were operated on within one day after admission. In other cases, the operation was delayed improving the preoperative state of the patient, for example by transfusion. The implementation of a hip replacement took an average of 45 minutes ( $35 \mathrm{~min}$ - 1h30). The approach was Hardingetype lateral in all patients. The femoral implant was cemented in 18 of our patients with standard stems (62\%), 3 recovery rods and a cementless resumption rod in a young patient. The acetabular insert was 18 times a cemented double mobility cup (80\%) and 2 times a cemented retentative cup to avoid the risk of postoperative dislocation (retentive cup with retention ring type Lépine $®$ ), and 02 standard cup.

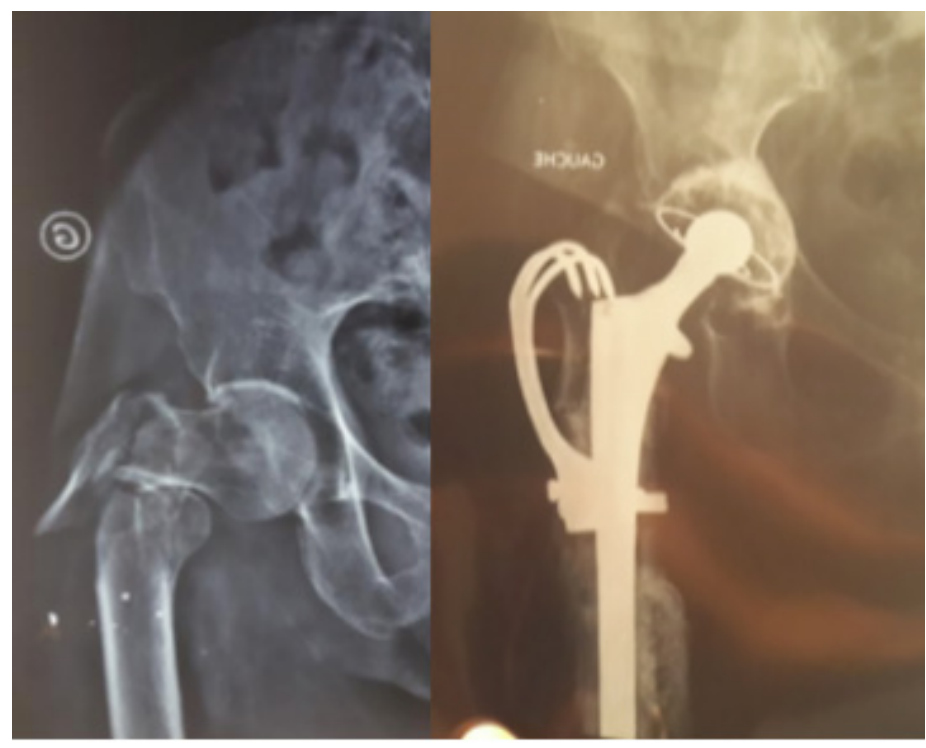

Figure 1: First-line prosthesis.

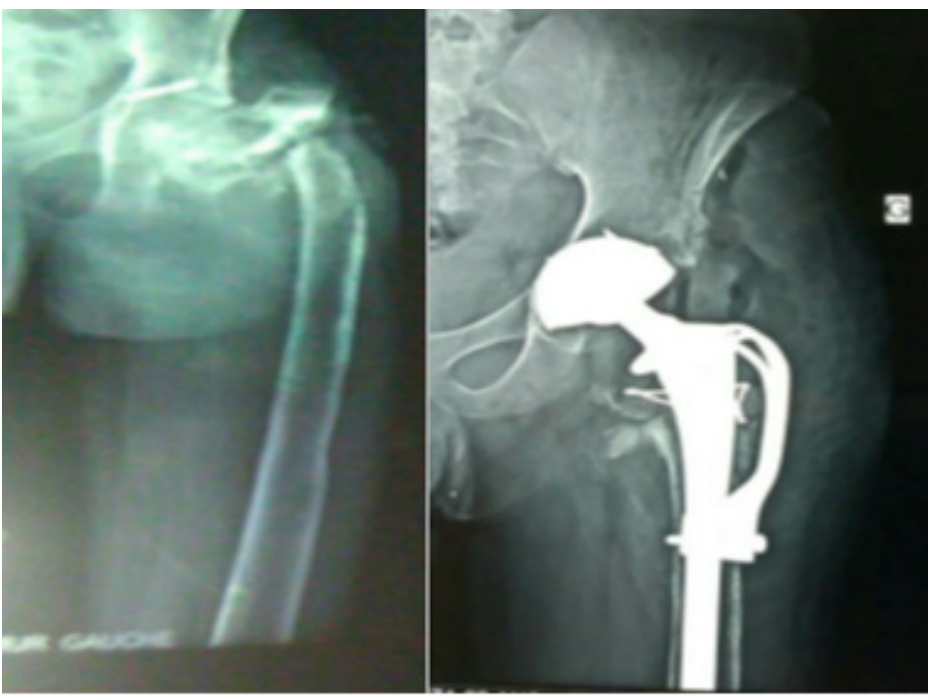

Figure 2: Failure of conservative treatment. 


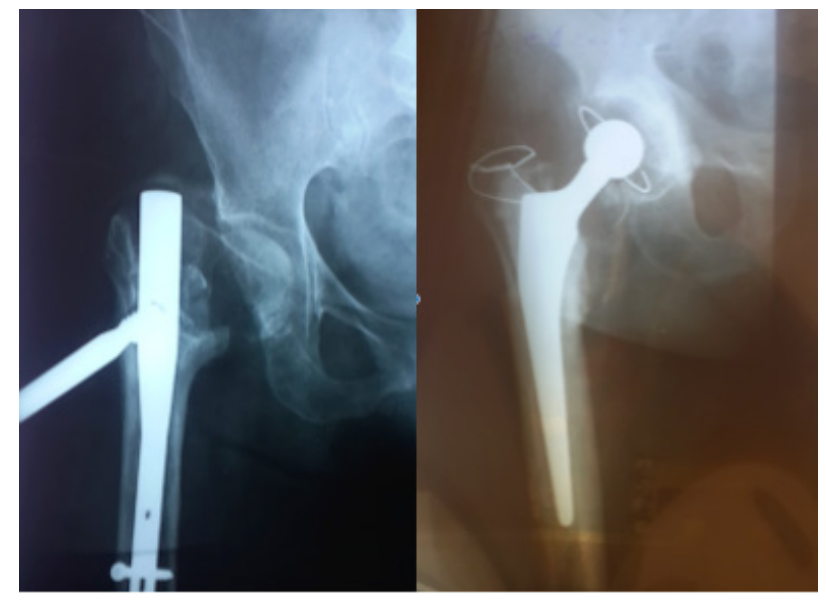

Figure 3: Disassembly of the osteosynthesis.

Table 1: The comorbidities.

\begin{tabular}{|c|c|}
\hline Comorbidites & Nombre \\
\hline Cardiaques & 14 \\
\hline Vasculaires & 7 \\
\hline Pneumologiques & 2 \\
\hline Renales & 1 \\
\hline Coxaethrose & 7 \\
\hline Gonarthrose & 3 \\
\hline
\end{tabular}

The diameter of the femoral head in this retentive cup was 22 or $28 \mathrm{~mm}$ depending on the outside diameter of the retentive cup. This retentive cup was made of polyethylene and sealed directly into the bone, without metal reinforcement. A trochanteric hook was placed in 9 cases. All patients received postoperative anticoagulation, either with antivitamin $\mathrm{K}$ or low molecular weight heparin, for a period of at least one month.

\section{Results}

At the last follow-up of 08 months on average (06 months-02 years), 2 patients died within 6 months postoperatively, one in each group. There were two dislocations (1.9\%) in patients with standard cup in the secondary prosthesis group after failure of conservative treatment. These episodes of luxation were repeated and required a reintervention for the installation of a retentive cup that solved the problem. No postoperative dislocation occurred in patients who had a retentive or dual mobility cup initially. No cases of sepsis were found. On review, the mean Parker score was 5.8 points for the first group and 5.2 for the second group (range 3 and 7). The mean PMA score was 12.6 points for the first group and 11.8 for the second (range 11-14). The loading was effective at 18.3 days on average and the recovery of the walking distance to 45 days. All the patients have started again. Half of the patients kept an English cane, mostly for a reason other than orthopedic (age, insecurity to walk). The most common orthopedic problems postoperatively were unequal length of the lower limbs (between $1 \mathrm{~cm}$ and $2 \mathrm{~cm}$ for all patients). The majority of patients had gluteal insufficiency, lameness, palpation pains in the trochanteric region, but none of these patients complained spontaneously.

\section{Discussion}

The ideal treatment for trochanteric fractures is still under debate as none of the existing osteosynthesis devices has been able to prove superiority in previous studies $[4,5]$. Geiger uses a standard cemented stem and a standard or dual mobility cemented cup. Since this process has demonstrated superiority to older internal fixation methods for these fractures, it has become the treatment of choice since 1992 [6]. The Mundada study [2] allowed us to compare the results of treatment of unstable trochanteric fractures treated with osteosynthesis and arthroplasty. All of our prosthesis-operated patients initially walked at 10 days on average. As for those reoperated after failure, the average duration of loading is longer than 27 days. No infections have been reported. Lyman et al. [4] noted the weight of the procedure and the less favorable clinical outcome after fracture arthroplasty, compared to a paired set of total hip arthroplasty prostheses.

Dobbs et al. [7] reported identical postoperative global mortality after arthroplasty and osteosynthesis but higher perioperatively due to more frequent cardiorespiratory complications after prosthesis. In our study, there were 2 deaths in the 6 months postoperative. Mortality was primarily influenced by patient-related problems, such as gender, age and comorbidities, but not the type of fracture. Consistent with previous studies [8] that confirm that the sum of four or more comorbidities increases the risk of dying by approximately $78 \%$. Dislocation is the major complication to be feared in patients treated with total hip replacement after unstable trochanteric fractures. It can be attributed to insufficient muscle stability, fractures of the greater or less trochanter. These notions have not been confirmed by Geiger et al. [3,6] who also underlined the high frequency of disassembly after screw plate or locked nail, more than $10 \%$, identical to the dislocation rate after arthroplasty. But this mechanical complication disappears when the cotyloid implant is of dual mobility type. The use of dual mobility prostheses may reduce this complication to an acceptable level $[1,5]$. We found 2 cases of dislocation in patients who did not have a dual mobility prosthesis or retentive cups. Osteosynthesis should be used for stable A1 and A2.1 fracture (AO classification) [9]. While primary arthroplasty is a viable option for the treatment of trochanteric 
fractures in previously independent autonomic patients, especially if osteoporosis prevents fixation, or in the presence of osteoarthritis [10-15].

\section{Conclusion}

Total hip arthroplasty is a reliable technique for treating the complications of osteosynthesis of per and subtrochanteric fractures in the elderly. It allowed the majority of these patients to return to a satisfactory function and walk that they had lost as a result of their trochanteric fracture. The complexity of the fracture associated with other factors related to the patient himself may lead the surgeon to consider arthroplasty as the treatment of choice. The use of dual mobility prostheses may reduce this complication to an acceptable level.

\section{References}

1. Haentjens P, Casteleyn PP, Opdecam P (1994) Primary bipolar arthroplasty or total hip arthroplasty for the treatment of unstable intertrochanteric and subtrochanteric fractures in elderly patients. Acta Orthop Belg 60(Suppl 1): 124-128.

2. Murdada A, Patil P, Gunki R (2013) Prospective study to compare communicated osteoporotic traumatic intertrochanteric femur fracture treated with primary cemented modular bipolar hemiarthroplasty with dynamic hip screw retrospectively. International Journal of Recent Trends in Science and Technology 9(2): 164-167.

3. Geiger F, Zimmerman-Stenzel M, Heisel C, Lehner B, Daecke W (2007) Trochanteric fracture in the elderly: the influence of primary hip arthroplasty on 1-year mortality. Arch Orthop Trauma Surg 127(10): 959-966.

4. Lyman JR, Kelley SS, Lachiewicz PF (2004) Hip arthroplasty after extra capsular hip fracture: a matched pair cohort analysis. J Surg Orthop Adv 13(1): 38-41.

5. Rodop O, Kiral A, Kaplan H, Akmaz I (2002) Primary bipolar hemi prosthesis for unstable intertrochanteric fractures. Int Orthop 26(4): 233-237.
6. Geiger F, Schreiner K, Schneider S, Pauschert R, Thomsen M (2006) Proximal fracture of the femur in elderly patients: The influence of surgical care and patient characteristics on post-operative mortality. Orthopade 35(6): 651-658.

7. Dobbs RE, Parvizi J, Lewallen DG (2005) Perioperative morbidity and 30-day mortality after intertrochanteric hip fractures treated by internal fixation or arthroplasty. J Arthroplasty 20(8): 963-968.

8. Kenzora JE, McCarthy RE, Lowell JD, Sledge CB (1984) Hip fracture mortality. Relation to age, treatment, preoperative illness, time of surgery, and complications. Clin Orthop Relat Res 186: 45-56.

9. Utrilla AL, Reig JS, Munoz FM, Tufanisco CB (2005) Trochanteric gamma nail and compression hip screw for trochanteric fractures: a randomized, prospective, comparative study in 210 elderly patients with a new design of the gamma nail. J Orthop Trauma 19(4): 229-233.

10. Waddell JP, Morton J, Schemitsch EH (2004) The role of total hip replacement in intertrochanteric fractures of the femur. Clin Orthop Relat Res 429: 49-53.

11. Hoffmann M, Hartel M, Rueger JM, Lehmann W (2014) Primary prosthetic replacement in per- and intertrochanteric fractures. Eur J Trauma Emerg Surg 40(3): 273-277.

12. Jones HW, Johnston P, Parker M (2006) Are short femoral nails superior to the sliding hip screw? A meta-analysis of 24 studies involving 3, 279 fractures. Int Orthop 30(2): 69-78.

13. Kim SY, Kim YG, Hwang JK (2005) Cementless calcar-replacement hemiarthroplasty compared with intramedullary fixation of unstable intertrochanteric fractures: A prospective, randomized study. J Bone Joint Surg Am 87(10): 2186-2192.

14. Lu-Yao GL, Baron JA, Barrett JA, Fisher ES (1994) Treatment and survival among elderly Americans with hip fractures: A population-based study. Am J Public Health 84(8): 1287-1291.

15. Hernigou P, Poignard A, Mathieu G, Cohen G, Manicom O, et al. (2006) Prothèses totales de hanche après échec de fixation de fractures per et sous-trochantériennes chez les sujets âgés. Revue de Chirurgie Orthopédique et Réparatrice de l'Appareil Moteur 92(4): 310-315. 